\title{
MEDIÇÃO DA QUALIDADE EM CENTRO CIRÚRGICO: QUAIS INDICADORES UTILIZAMOS?
}

\author{
Quality measurement in the operating room: which indicators do we use?
}

Medida de calidad en centro quirúrgico: ¿qué indicadores usamos?

\author{
Bárbara Peres Gama ${ }^{1 *}$ (), Elena Bohomol ${ }^{2}$ (])
}

RESUMO: Objetivo: Identificar quais são e como são gerenciados os indicadores utilizados pelos enfermeiros que atuam em centro cirúrgico. Método: Estudo descritivo, transversal e quantitativo, realizado no período de outubro de 2018 a fevereiro de 2019. A amostra foi composta de conveniência, com enfermeiros de centros cirúrgicos brasileiros que responderam a um questionário com 53 questões. A análise de dados foi realizada por meio de estatística descritiva e teste estatístico $\chi^{2}$, com nível de significância de $5 \%$. Resultados: Os indicadores mais gerenciados pelos enfermeiros foram: quantidade de cirurgias canceladas (81,6\%), taxa de infecção do sítio cirúrgico $(78,5 \%)$ e ocupação de salas cirúrgicas por mês (69,6\%). Observou-se diferença significante da gestão dos indicadores entre hospitais com e sem avaliação externa de qualidade, nos quesitos treinamentos $(p=0,000)$ e compartilhamento dos resultados com as equipes ( $\mathrm{p}=0,000)$, que se mostraram iguais para dificuldade em utilizar a ferramenta $(\mathrm{p}=0,803)$. Conclusão: Apesar de os indicadores serem monitorados pelos enfermeiros e os hospitais com avaliação externa apresentarem melhores resultados em alguns itens de gerenciamento e uso de indicadores, as instituições ainda precisam investir no aprimoramento dos profissionais e na gestão da ferramenta.

Palavras-chave: Enfermagem. Centros cirúrgicos. Indicadores de gestão. Gestão da qualidade. Segurança do paciente.

ABSTRACT: Objective: To identify the indicators used by nurses working in the operating room and how they are managed. Method: Descriptive, cross-sectional, and quantitative study, carried out from October 2018 to February 2019. The sample was a convenience sample, with nurses from Brazilian operating rooms who answered a questionnaire with 53 questions. Data analysis was performed using descriptive statistics and $\chi^{2}$ statistical test with a significance level of $5 \%$. Results: The indicators most managed by nurses were: number of surgeries canceled (81.6\%), infection rate of the surgical site (78.5\%), and occupation of operating rooms per month (69.6\%). There was a significant difference in the management of indicators between hospitals with and without external quality assessment, in terms of training $(\mathrm{p}=0.000)$ and sharing the results with the teams $(\mathrm{p}=0.000)$, which proved to be equal for the difficulty in using the tool ( $\mathrm{p}=0.803$ ). Conclusion: Although the indicators are monitored by nurses and hospitals with external assessment show better results in some items of management and use of indicators, institutions still need to invest in the improvement of professionals and the management of the tool.

Keywords: Nursing. Operating rooms. Management indicators. Quality management. Patient safety.

RESUMEN: Objetivo: identificar los indicadores utilizados por las enfermeras que trabajan en el quirófano y cómo se gestionan. Método: Estudio descriptivo, transversal y cuantitativo, realizado entre octubre de 2018 y febrero de 2019. La muestra fue por conveniencia, compuesta por enfermeras de centros quirúrgicos brasileños que respondieron un cuestionario con 53 preguntas. El análisis de los datos se realizó mediante estadística descriptiva y prueba estadística de $\chi^{2}$, con un nivel de significación del $5 \%$. Resultados: Los indicadores más manejados por las enfermeras fueron: número de cirugías canceladas $(81,6 \%)$, tasa de infección del sitio quirúrgico (78,5\%) y ocupación de quirófanos por mes (69,6\%). Hubo una diferencia significativa en el manejo de los indicadores entre hospitales con y sin evaluación de calidad externa, en términos de capacitación ( $\mathrm{p}=0,000)$ y el intercambio de resultados con los equipos ( $\mathrm{p}=0,000)$, mostrando lo mismo para la dificultad en el uso de la herramienta $(\mathrm{p}=0,803)$. Conclusión: Aunque los indicadores son monitoreados por enfermeras y hospitales con evaluación externa, muestran mejores resultados en algunos ítems de gestión y uso de indicadores, las instituciones aún necesitan invertir en la mejora de los profesionales y en el manejo de la herramienta.

Palabras clave: Enfermería. Centros quirúrgicos. Indicadores de gestión. Gestión de la calidad. Seguridad del paciente.

'Enfermeira pela Escola Paulista de Enfermagem da Universidade Federal de São Paulo. Enfermeira do Hospital Universitário da Universidade Federal de São Paulo- São Paulo (SP), Brasil.

${ }^{2}$ Livre-docente do Departamento de Orientação Profissional da Escola de Enfermagem da Universidade de São Paulo - São Paulo (SP), Brasil.

*Autora correspondente: barbaraperesgama94@gmail.com

Recebido: 17/12/2019 - Aprovado: 17/07/2020

https://doi.org/10.5327/Z1414-4425202000030004 


\section{INTRODUÇÃO}

Com o avanço da saúde e a sistematização do cuidado, a criação de medidas técnicas de mensuração de dados, baseadas em evidências, fiáveis e de qualidade, foi essencial para abranger de forma eficiente todas as áreas que envolvem a saúde ${ }^{1}$.

Os indicadores são ferramentas utilizadas para analisar, adquirir, identificar e mensurar ações ou informações relativas à qualidade de atendimento, doença, epidemiologia e saúde dos contextos abordados, servindo para sintetizá-los por meio de conceitos numéricos. Sua elaboração depende do que se quer investigar, segundo as necessidades individuais de cada situação. A qualidade das informações utilizadas resultará em sua eficácia e confiabilidade ${ }^{1,2}$.

Os diferentes processos assistenciais, as implicações logísticas e a vulnerabilidade do paciente demandam acompanhamento de medições padronizadas e estruturadas ao longo do tempo, para que a avaliação do desempenho e a adequação estrutural proporcionem condições de conforto e segurança tanto para o paciente quanto para a equipe. Os indicadores são capazes de sinalizar desvios e permitem a revisão do problema, impedindo que essas situações se tornem rotineiras ${ }^{3,4}$.

Ao utilizar os indicadores, devem-se levar em conta aqueles que sejam capazes de se adequar às características e singularidades do setor. O centro cirúrgico (CC) é uma unidade complexa, com demanda de assistência espontânea e emergencial e especificidades, que necessita de controle sobre seu funcionamento a fim de prestar atendimento de qualidade. $\mathrm{O}$ uso de indicadores concede aos enfermeiros um norteamento para a gestão do setor, viabiliza o controle dos processos, garante a qualidade dos serviços, além de gerir sua série histórica, avaliando os processos de melhoria implantados ${ }^{3,5}$.

Dessa maneira, os riscos e as consequências negativas podem ser evitados dentro do CC, se a utilização de indicadores considerados de qualidade e o monitoramento da gestão forem eficientes ${ }^{3}$. Instituições que têm processos avaliativos contínuos, tais como programas de certificação ou acreditação, são mais preparadas para realizar atendimento qualificado e lidam com mais facilidade com o gerenciamento de seus indicadores, conseguindo manter seus resultados com maior invariabilidade ${ }^{6,7}$.

Diferentes indicadores são propostos para gestão de um CC, como a ocorrência de lesão por posicionamento, lesão de pele, queda, queimadura por bisturi elétrico, manutenção da normotermia nos pacientes e tempo de rotatividade das salas cirúrgicas, entre outros, a depender da instituição, do envolvimento dos profissionais e das cobranças da alta direção $0^{3-5}$. Levando em consideração de que nem todos os estabelecimentos utilizam indicadores para a gestão do CC e que também não existe consenso sobre quais são necessários para o monitoramento da qualidade nesse serviço, realizou-se a presente pesquisa. Assim, pelos resultados encontrados, as autoras se propõem a responder à seguinte pergunta: quais indicadores são utilizados pelos enfermeiros para a gestão de um CC?

\section{OBJETIVO}

Identificar quais são e como são gerenciados os indicadores utilizados pelos enfermeiros que atuam em CC.

\section{MÉTODO}

Estudo descritivo, transversal, com abordagem quantitativa, realizado no período de 10 de outubro de 2018 a 10 de fevereiro de 2019. A amostra foi definida por conveniência, com população de profissionais associados à Sociedade Brasileira de Enfermeiros de Centro Cirúrgico, Recuperação Anestésica e Centro de Material e Esterilização (SOBECC), que podem ser enfermeiros, técnicos de enfermagem, auxiliares de enfermagem, estudantes, instrumentadores ou outros interessados.

Elaborou-se um instrumento estruturado, baseado em publicações científicas sobre indicadores e gerenciado pelo programa Research Electronic Data Capture (REDCap), de forma on-line. O instrumento foi composto de duas partes: a primeira com nove questões para a caracterização sociodemográfica e profissional dos participantes e a segunda com 42 questões fechadas sobre indicadores e gerenciamento, com as opções de resposta sim ou não, além de duas questões abertas. Como parte das questões, foram elencados 33 indicadores mencionados na literatura científica como possíveis de serem utilizados ${ }^{2,7}$. Realizou-se um pré-teste do instrumento com quatro voluntários atuantes na área de CC, que apontaram melhorias na sua estrutura, solicitaram esclarecimentos nas questões ambíguas e sugeriram acréscimo de indicadores, que foram acatados. Com o pré-teste foi possível estimar o tempo de 20 min para o preenchimento da pesquisa.

Obedeceu-se à diretriz da Sociedade em relação à distribuição do questionário, cujas mensagens aos profissionais cadastrados em nível nacional foram gerenciadas pela secretaria da SOBECC, por meio de um link de acesso, precedido do Termo de Consentimento Livre e Esclarecido. Foram enviadas três mensagens de lembretes aos associados durante o período de coleta de dados. O critério de exclusão adotado foi o de profissionais que 
não eram enfermeiros, uma vez que há sócios de outras categorias profissionais na sociedade, como citado anteriormente.

Os dados obtidos foram organizados em planilha Excel $^{\circledR}$ e analisados por meio de estatística descritiva e teste estatístico $\chi^{2}$, utilizando nível de significância de $5 \%(\mathrm{p}<0,05)$.

O estudo foi aprovado pelo Comitê de Ética em Pesquisa da Universidade Federal de São Paulo, sob Certificado de Apresentação para Apreciação Ética (CAAE) $n^{\circ}$ 68023617.0.0000.5505, e pela diretoria da SOBECC.

\section{RESULTADOS}

Responderam à pesquisa 162 pessoas e quatro questionários foram excluídos por não se tratar de enfermeiros. Assim sendo, a amostra foi composta de 158 enfermeiros, sendo 94 (59,5\%) especialistas em diversas áreas como CC, Urgência e Emergência e Gestão em Saúde; 81 (51,3\%) em cargos assistenciais e $77(48,7 \%)$ em cargos gerenciais; 58 (36,7\%) com média de atuação acima de 10 anos, sendo o tempo médio de todos os participantes de 10,3 anos; 57 (36,1\%) provenientes do estado de São Paulo, 21 (13,3\%) do Rio de Janeiro e 17 (10,8\%) do Rio Grande do Sul; $66(41,8 \%)$ trabalhavam em hospitais privados e, destes, 70 (44,3\%) em hospitais especializados; 53 (33,5\%) enfermeiros trabalhavam em hospitais de grande porte (com mais de 300 leitos) e 81 (47,5\%) em hospitais com certificação externa de avaliação da qualidade.

Apresentaram-se 33 indicadores, e os participantes apontaram sua utilização no serviço. Todos os indicadores são mensurados em maior ou menor frequência e 10 deles eram gerenciados por mais de $50 \%$ dos profissionais. Entre as citações consideradas menos recorrentes, evidenciaram-se indicadores gerenciados por menos de $25 \%$, como pode ser observado na Tabela 1.

Ao serem perguntados se gerenciavam outros indicadores que não foram contemplados pela pesquisa, 27 (17,1\%) enfermeiros responderam que sim e destacaram: número de cirurgias robóticas por mês, tempo de giro de sala, atraso médico, monitoramento do tempo em que a sala operatória (SO) ficou parada esperando a chegada da equipe médica, número de cirurgias que ultrapassaram o tempo programado no agendamento, lesão por uso de garrote pneumático e taxa de efetividade de checklist de cirurgia segura.

Perguntou-se aos participantes se implantariam outros indicadores além dos que foram apresentados e $46(29,1 \%)$ referiram que sim, destacando: agendamento cirúrgico errado ou incorreto, número de cirurgias por profissional, tempo de permanência

Tabela 1. Indicadores utilizados pelos enfermeiros dos centros cirúrgicos de hospitais brasileiros.

\begin{tabular}{|c|c|c|}
\hline Indicadores & n & $\%$ \\
\hline Quantidade de cirurgias canceladas & 129 & 81,6 \\
\hline Infecção de sítio cirúrgico & 124 & 78,5 \\
\hline Ocupação de salas cirúrgicas por mês & 110 & 69,6 \\
\hline Adesão à lista de verificação de cirurgia segura & 103 & 65,2 \\
\hline $\begin{array}{l}\text { Pacientes com antibioticoprofilaxia no momento } \\
\text { adequado }\end{array}$ & 96 & 60,7 \\
\hline Acidentes de trabalho de profissionais de enfermagem & 95 & 60,1 \\
\hline Quedas & 94 & 59,5 \\
\hline Absenteísmo de profissionais de enfermagem & 88 & 55,7 \\
\hline Lesões de pele & 86 & 54,4 \\
\hline $\begin{array}{l}\text { Quantidade de cirurgias realizadas sem } \\
\text { programação prévia }\end{array}$ & 82 & 51,9 \\
\hline Treinamento de profissionais de enfermagem & 78 & 49,4 \\
\hline Quantidade de cirurgias realizadas por dia & 72 & 45,6 \\
\hline $\begin{array}{l}\text { Disponibilidade de equipamentos para realização } \\
\text { de cirurgia }\end{array}$ & 70 & 44,3 \\
\hline $\begin{array}{l}\text { Sistematização da assistência de enfermagem } \\
\text { perioperatória }\end{array}$ & 68 & 43,0 \\
\hline Erros de administração de medicação & 66 & 41,8 \\
\hline $\begin{array}{l}\text { Tempo médio de permanência na recuperação } \\
\text { pós-anestésica }\end{array}$ & 66 & 41,8 \\
\hline Lesão por posicionamento cirúrgico & 65 & 41,1 \\
\hline Completude de preenchimento de prontuário & 65 & 41,1 \\
\hline Tempo médio de permanência na sala de cirurgia & 65 & 41,1 \\
\hline Cirurgias em local errado & 64 & 40,5 \\
\hline Queimadura de pele por bisturi elétrico & 64 & 40,5 \\
\hline $\begin{array}{l}\text { Rotatividade de profissionais de enfermagem } \\
\text { (turnover) }\end{array}$ & 59 & 37,4 \\
\hline $\begin{array}{l}\text { Disponibilidade de serviços (laboratório, radiologia, } \\
\text { outros) }\end{array}$ & 58 & 36,7 \\
\hline Cirurgias em paciente errado & 57 & 36,0 \\
\hline Atraso de cirurgia & 56 & 35,5 \\
\hline Complicações após sedação & 55 & 34,8 \\
\hline $\begin{array}{l}\text { Mortalidade cirúrgica intra-hospitalar ajustada ao } \\
\text { risco }\end{array}$ & 55 & 34,8 \\
\hline Retorno não programado à sala cirúrgica & 51 & 32,3 \\
\hline $\begin{array}{l}\text { Presença do enfermeiro durante todo o período de } \\
\text { funcionamento }\end{array}$ & 51 & 32,3 \\
\hline Pacientes com manutenção da normotermia & 44 & 27,8 \\
\hline Pacientes com abreviação de jejum & 33 & 20,9 \\
\hline Enfermeiros com título de especialista & 33 & 20,9 \\
\hline Participação de enfermeiros em eventos científicos & 30 & 19,0 \\
\hline
\end{tabular}


do paciente no $\mathrm{CC}$ antes do encaminhamento à $\mathrm{SO}$, visita pré-anestésica, visita de enfermagem, reação adversa em transfusões sanguíneas e contagem de instrumentais (disponibilizados versus devolvidos para Centro de Material e Esterilização).

A Tabela 2 apresenta a comparação entre os indicadores gerenciados por mais de $50 \%$ dos participantes, segundo os hospitais que têm ou não têm processos avaliativos continuados. Os resultados mostram que, de 10 indicadores, oito têm diferença significante, portanto há mais monitoramento de indicadores em instituições que trabalham sua gestão da qualidade, submetendo-se a processos avaliativos externos.

Quanto ao gerenciamento dos indicadores, a maioria dos participantes referiu que eles expressam a realidade vivenciada
(120/80,5\%), são analisados pelos gestores/responsáveis (128/85,9\%), os resultados são discutidos com a equipe de enfermagem $(90 / 60,4 \%)$, os processos de melhoria são implementados com base nos resultados $(105 / 70,5 \%)$ e existe uma série histórica dos indicadores $(97 / 65,1 \%)$. No entanto a maioria respondeu negativamente às questões sobre a coleta de indicadores ser um processo simples $(92 / 61,7 \%)$, os resultados serem discutidos com a equipe multiprofissional (77/51,7\%), os resultados serem comparados com os de outras instituições $(108 / 72,5 \%)$ e haver treinamento para gerenciá-los $(97 / 65,1 \%)$, conforme a Tabela 3.

A Tabela 4 apresenta a comparação entre o gerenciamento dos indicadores entre os hospitais que têm ou não

Tabela 2. Comparação entre os indicadores gerenciados por mais de $50 \%$ dos participantes segundo hospitais que têm ou não processos avaliativos continuados.

\begin{tabular}{|l|c|c|c|c|c|}
\hline Indicadores & \multicolumn{2}{|c|}{$\begin{array}{c}\text { Com avaliação } \\
\text { n=71 }\end{array}$} & $\begin{array}{c}\text { Sem avaliação } \\
\text { n=84 }\end{array}$ & $\mathbf{p}^{*}$ \\
\hline Quantidade de cirurgias canceladas & 63 & 88,7 & 64 & 76,2 & 0,043 \\
\hline Infecção de sítio cirúrgico & 65 & 91,5 & 57 & 67,8 & 0,000 \\
\hline Ocupação de salas cirúrgicas por mês & 59 & 83,0 & 51 & 60,7 & 0,002 \\
\hline Adesão à lista de verificação de cirurgia segura & 59 & 83,0 & 44 & 52,4 & 0,000 \\
\hline Pacientes que receberam antibioticoprofilaxia no momento adequado & 56 & 78,9 & 38 & 45,2 & 0,000 \\
\hline Acidente de trabalho de profissionais de enfermagem & 47 & 66,2 & 47 & 55,9 & 0,193 \\
\hline Queda & 52 & 73,2 & 40 & 47,6 & 0,001 \\
\hline Absenteísmo de profissionais de enfermagem & 44 & 62,0 & 43 & 51,2 & 0,177 \\
\hline Lesão de pele & 48 & 67,6 & 38 & 45,2 & 0,005 \\
\hline Quantidade de cirurgias realizadas sem programação prévia & 44 & 62,0 & 37 & 44,0 & 0,03 \\
\hline
\end{tabular}

*teste do $\chi^{2}(0,05)$.

Tabela 3. Itens relacionados ao gerenciamento dos indicadores.

\begin{tabular}{|c|c|c|c|c|c|}
\hline \multirow{2}{*}{ Itens de gerenciamento } & \multicolumn{2}{|c|}{ Sim } & \multicolumn{2}{|c|}{ Não } & \multirow{2}{*}{ Total } \\
\hline & $n$ & $\%$ & $n$ & $\%$ & \\
\hline $\begin{array}{l}\text { Os indicadores que você utiliza expressam a realidade institucional na qual você } \\
\text { trabalha? }\end{array}$ & 120 & 80,5 & 26 & 17,5 & 146 \\
\hline Você julga a coleta de indicadores um processo simples? & 54 & 36,2 & 92 & 61,7 & 146 \\
\hline Os indicadores são analisados pelos gestores/responsáveis? & 128 & 85,9 & 19 & 12,7 & 147 \\
\hline Os resultados são discutidos com a equipe de enfermagem? & 90 & 60,4 & 57 & 38,2 & 147 \\
\hline Os resultados são discutidos com a equipe multiprofissional? & 67 & 45,0 & 77 & 51,7 & 144 \\
\hline Os processos de melhoria são implementados com base nos indicadores? & 105 & 70,5 & 40 & 26,8 & 145 \\
\hline $\begin{array}{l}\text { Existe uma série histórica dos indicadores que propicie visão dos resultados ao longo } \\
\text { do tempo? }\end{array}$ & 97 & 65,1 & 46 & 30,9 & 143 \\
\hline Os resultados são comparados com os de outras instituições? & 39 & 26,2 & 108 & 72,5 & 147 \\
\hline Você teve treinamento para gerenciar os indicadores? & 51 & 34,2 & 97 & 65,1 & 148 \\
\hline
\end{tabular}


Tabela 4. Comparação dos Itens de gerenciamento com respostas “sim” para hospitais com e sem avaliação externa.

\begin{tabular}{|c|c|c|c|c|c|}
\hline \multirow{2}{*}{$\begin{array}{l}\text { Itens de gerenciamento } \\
\text { Os indicadores que você utiliza expressam a realidade } \\
\text { institucional na qual você trabalha? }\end{array}$} & \multicolumn{2}{|c|}{$\begin{array}{c}\text { Com avaliação } \\
n=71\end{array}$} & \multicolumn{2}{|c|}{$\begin{array}{l}\text { Sem avaliação } \\
n=84\end{array}$} & \multirow{2}{*}{$\begin{array}{l}\text { Valor } p^{*} \\
0,12\end{array}$} \\
\hline & 59 & 83,1 & 61 & 72,6 & \\
\hline Você julga a coleta de indicadores um processo simples? & 24 & 33,8 & 30 & 35,7 & 0,803 \\
\hline Os indicadores são analisados pelos gestores/responsáveis? & 63 & 88,7 & 65 & 77,4 & 0,63 \\
\hline Os resultados são discutidos com a equipe de enfermagem? & 52 & 73,2 & 38 & 45,2 & 0,000 \\
\hline Os resultados são discutidos com a equipe multiprofissional? & 41 & 57,7 & 26 & 30,9 & 0,000 \\
\hline $\begin{array}{l}\text { Os processos de melhoria são implementados com base nos } \\
\text { indicadores? }\end{array}$ & 54 & 76,0 & 51 & 60,7 & 0,041 \\
\hline $\begin{array}{l}\text { Existe uma série histórica dos indicadores que propicie } \\
\text { visão dos resultados ao longo do tempo? }\end{array}$ & 56 & 78,9 & 41 & 48,8 & 0,000 \\
\hline Os resultados são comparados com os de outras instituições? & 22 & 31,0 & 17 & 20,2 & 0,124 \\
\hline Você teve treinamento para gerenciar os indicadores? & 33 & 46,5 & 18 & 21,4 & 0,000 \\
\hline
\end{tabular}

têm processos avaliativos continuados. Mesmo não havendo diferença significante, os profissionais de ambos os hospitais acreditam que o processo de coleta de indicadores não é simples $(\mathrm{p}=0,803)$. O que se verifica é o baixo investimento no treinamento dos enfermeiros nos dois tipos de instituição, mas os que possuem processos avaliativos ainda o têm em maior frequência do que aqueles que não possuem $(\mathrm{p}=0,000)$.

Entre essas questões, ainda há quatro outras que apresentaram diferença significante, apontando que há mais envolvimento das equipes de enfermagem e multiprofissionais na discussão dos resultados $(\mathrm{p}=0,000$ e $\mathrm{p}=0,000$, respectivamente), análise ao longo do tempo da ferramenta $(\mathrm{p}=0,000)$ e maior preocupação com as melhorias baseadas nos indicadores $(p=0,041)$ em instituições submetidas a processos avaliativos externos.

\section{DISCUSSÃO}

A pesquisa permitiu conhecer os enfermeiros atuantes em CC e suas instituições, bem como quais são os indicadores utilizados. Um dos dados que se destacam é a maioria de os profissionais ter título de especialista e ocupar cargo assistencial, o que permite identificar um grupo de profissionais preparados para atuar em prol do paciente em um setor com tamanha complexidade. Estudo realizado em sete hospitais públicos e privados em duas cidades brasileiras para avaliar a percepção do enfermeiro acerca de sua importância em relação ao trabalho e à gestão do setor apontou uma população de participantes majoritariamente de especialistas (80\%), fato que faz refletir sobre a diferenciação no preparo profissional para a atuação na área ${ }^{8}$. No entanto nossa pesquisa evidenciou que ter a titulação de especialista não é um indicador frequentemente monitorado pelas instituições.

Outro estudo nacional, realizado em um hospital filantrópico de Minas Gerais para compreender a percepção dos profissionais de enfermagem com relação aos empecilhos presentes durante a assistência no período intraoperatório, apresentou uma estrutura organizacional com enfermeiros gerentes, supervisores e em maior parte assistenciais, revelando a atuação diferenciada de trabalho deles em comparação ao corpo de técnicos de enfermagem ${ }^{9}$.

O grupo de profissionais na presente pesquisa apontou para o monitoramento de um leque de indicadores, como o observado nos resultados. No entanto, estudos sugerem que os indicadores não devem ser estanques, podendo ser modificados com base nas necessidades do paciente ou dos serviços. Em uma revisão de literatura, os autores citaram como importantes os indicadores voltados ao monitoramento de lesões, quedas, queimaduras por bisturi elétrico, lesões por posicionamento e infecção do sítio cirúrgico ${ }^{3}$. Outra publicação sugeriu indicadores como tempo operatório, primeira cirurgia do dia e tempo não operatório, relacionados à gestão do tempo das cirurgias, à disponibilidade de materiais e ao preparo de $\mathrm{SO}^{10}$. 
No entanto chamam a atenção esses mesmos indicadores, que deveriam ser monitorados em sua completude, por exemplo, o de infecção relacionada à assistência à saúde. Conforme a Portaria n ${ }^{\circ}$ 2.616/1998, do Ministério da Saúde, cabe, obrigatoriamente, a todas as instituições prevenir e monitorar indicadores referentes às taxas de infecção, para que não haja prejuízo aos pacientes ${ }^{11}$.

Outro indicador é a adesão à lista de cirurgia segura e o uso de antibioticoprofilaxia como parte do checklist da cirurgia segura. Desde 2013, o Programa Nacional de Segurança do Paciente apresenta inúmeras iniciativas para a implantação e o gerenciamento adequado desse protocolo, com indicadores específicos, porém ainda se verificam dificuldades nas instituições, tais como falha no processo de preenchimento, falta de adesão de toda a equipe e problemas com as informações relatadas ${ }^{12}$. Por outro lado, verifica-se o empenho em tentar melhorar a adesão a esse protocolo, por meio da mudança da cultura institucional, de educação continuada, treinamentos e auditorias internas ${ }^{13}$.

Um dos indicadores menos frequentes encontrados nesta pesquisa é o de abreviação de jejum. Sua importância se dá porque ela propicia melhor sensação durante o pré-operatório e ajuda na recuperação pós-cirúrgica, pois quanto maior o período de jejum maior será a resposta metabólica, aumentando a resposta hormonal relacionada ao processo inflamatório e influenciando o processo cicatricial ${ }^{14}$. Estudo destaca a importância de construir um ambiente agradável de atendimento em momentos de desconforto, além de prever a recuperação mais eficiente do paciente ${ }^{14}$.

Os indicadores citados pela literatura e considerados clássicos, como queda, lesão por posicionamento cirúrgico, queimadura por bisturi elétrico e lesão são vistos como fundamentais; no entanto, seu monitoramento é baixo no grupo estudado, revelando um resultado preocupante. Estudos mencionam a necessidade do comprometimento do enfermeiro para assegurar que danos não sejam causados ao paciente durante sua estadia no CC, evitar agravos em sua condição e prover recuperação adequada, não prolongando seu tempo no ambiente hospitalar ${ }^{3,15}$.

A pesquisa mostrou o dinamismo do monitoramento de indicadores baseados nas necessidades de cada serviço, razão pela qual eles devem ser continuamente avaliados. Essa ideia tornou-se evidente no momento em que os participantes citaram outros indicadores não contemplados pela pesquisa, como, por exemplo, o emprego da cirurgia robótica no ambiente cirúrgico. É esperado que esse indicador seja monitorado em poucos hospitais, uma vez que se trata de tecnologia diferenciada. Tal fato traz desafios no desenvolvimento de competências do enfermeiro em relação a esse tipo de cirurgia. Para que seja possível gerenciar esse indicador, deve-se estar a par de como o procedimento funciona, de quais são as necessidades do paciente, da equipe, de materiais e espaço físico, bem como da atuação da equipe de enfermagem ${ }^{16}$.

Embora não sejam maioria, verificou-se um contingente grande de profissionais que atuam em hospitais com certificação de qualidade. As instituições que se submetem a esses processos gerenciam mais os indicadores do que aquelas que não o fazem. Essa afirmativa é corroborada por estudo árabe que apresenta resultados em relação ao monitoramento de indicadores, impactando a melhoria do atendimento e os resultados ao paciente antes e depois da acreditação ${ }^{17}$.

A presente pesquisa trouxe a importância do gerenciamento dos indicadores e de seu conhecimento por todos os envolvidos. Torna-se evidente que usar corretamente a ferramenta e discutir seus resultados com as equipes gera maior empenho e compreensão sobre a assistência e os processos a serem instituídos para melhorias no atendimento. Por meio da gestão e da compreensão adequada da ferramenta, o enfermeiro será capaz de manter um controle mais bem estruturado das atividades, ao pontuar os aspectos de melhoria e os pontos positivos de seu serviço, o que os tornará mais claros e eficientes ${ }^{15}$.

O compartilhamento desses resultados com as equipes é essencial, uma vez que todos fazem parte do processo de assistência ao paciente, sendo o enfermeiro responsável em gerir o CC para que haja efetividade nas atividades realizadas ${ }^{18}$.

Os hospitais que possuem tal característica apresentam ambientes melhores de se trabalhar com relação ao atendimento dos pacientes e à disposição dos profissionais. Há incentivos constantes para se realizarem notificações de eventos adversos, de modo que melhorias sejam feitas no serviço. Além disso, existe mudança significativa nos registros, uma vez que há compreensão da importância do registro adequado ${ }^{19}$.

O aprendizado organizacional implica continuamente rever os processos e desenvolver uma liderança comprometida com a segurança do paciente, visto que ela fornece apoio contínuo para os esforços e iniciativas de melhorias nos diferentes níveis hierárquicos. A cultura hospitalar propõe um processo de notificação de eventos adversos e ações 
não punitivas para erros, para incentivar os profissionais a identificar falhas, comunicá-las e aprender com elas ao invés de culpabilizar-se, além de incentivar o trabalho em equipe, que, segundo estudo, se apresenta como questão ressaltada pelos enfermeiros como de extrema importância para realizar a assistência. Além disso, o processo de acreditação pode ajudar no desenvolvimento do aprendizado institucional, uma vez que influencia na melhora e no gerenciamento dos processos pela equipe e no empenho em estar em conformidade com o que é preconizado com relação à cultura de segurança do paciente ${ }^{12,20}$.

Todavia, novas pesquisas sobre o uso de indicadores em CC e sua gestão podem ser propostas, abordando como são os sistemas informacionais das instituições, como é o processo de notificação de eventos e a análise da causa-raiz, condições essenciais para a melhoria contínua da assistência ${ }^{21}$.

As limitações do estudo referem-se ao fato de que foram apresentadas informações com diferenças significantes sobre enfermeiros que trabalham em hospitais com processos avaliativos. No entanto não há como saber o número de hospitais representados por eles, o que pode ser um viés da presente pesquisa. Como opção metodológica, os indicadores foram apresentados por seu título e não por sua fórmula, o que pode ter gerado entendimentos diferentes nos participantes e influenciado suas respostas.

\section{CONCLUSÃO}

A realização deste estudo permitiu verificar quais e como os enfermeiros gerenciam os indicadores no CC, sendo todos os indicadores gerenciados em maior ou menor número. Quantidade de cirurgias canceladas, taxa de infecção do sítio cirúrgico, ocupação de salas cirúrgicas por mês, adesão à lista de verificação de cirurgia segura e pacientes que receberam antibioticoprofilaxia no momento adequado estão entre os indicadores mais monitorados. Em contrapartida, pacientes com abreviação de jejum, enfermeiros com título de especialista e participação de enfermeiros em eventos científicos são os indicadores menos monitorados.

Nota-se que os enfermeiros compreendem a necessidade da ferramenta, uma vez que outros indicadores, como cirurgia robótica, são citados como parte de sua realidade assistencial.

A gestão dos indicadores está presente, mais ainda é considerada um processo difícil pela maioria dos profissionais, não havendo diferença significante entre instituições com e sem avaliação externa da qualidade para esse item. Há falta de treinamento em ambos os tipos de hospitais, porém com diferença significante, já que instituições com acreditação possuem mais treinamentos para gerenciar a ferramenta, além de discutir seus resultados com a equipe de enfermagem e multiprofissional.

Percebe-se que as instituições avaliadas possuem resultados significantemente melhores em relação a alguns indicadores e itens de gerenciamento. Disso se pode inferir que estão mais bem preparadas para o atendimento, para criar melhorias baseadas em seus resultados, e mais próximas de atingir a excelência na assistência, uma vez que os indicadores permitem evitar desperdícios financeiros e materiais e proporcionam visão ampla do cuidado, reduzindo, dessa maneira, riscos e agravos aos pacientes.

\section{AGRADECIMENTOS}

Agradecemos ao Conselho Nacional de Desenvolvimento Científico e Tecnológico (CNPq), a concessão da bolsa de Iniciação em Desenvolvimento Tecnológico e Inovação (PIBITI) $n^{\circ} 122589$ / 2018-1, que nos proporcionou a oportunidade de realizar esta pesquisa por meio de seu financiamento.

\section{REFERÊNCIAS}

1. Organização Pan-Americana de Saúde (OPAS). Indicadores de salud: elementos básicos para el análisis de la situacion. Boletim Epidemiológico [Internet]. 2001 [access on 10/29/2019];22(4):116. Available at: http://www.paho.org/spanish/SHA/be_v22n4indicadores.htm

2. Oliveira JR, Xavier RMF, Santos Júnior AF. Eventos adversos notificados ao Sistema Nacional de Notificações para a Vigilância
Sanitária (NOTIVISA): Brasil, estudo descritivo no período 2006 a 2011. Epidemiol Serv Saúde. 2013;22(4):671-8. http://dx.doi.org/10.5123/ S1679-49742013000400013

3. Amaral JAB, Spiri WC, Bocchi SCM. Indicadores de qualidade em enfermagem com ênfase no centro cirúrgico: revisão integrativa da literatura. Rev SOBECC. 2017;22(1):42-51. http://dx.doi.org/10.5327/ Z1414-4425201700010008 
4. Duarte IG, Ferreira DP. Uso de indicadores na gestão de um centro cirúrgico. Rev Adm Saúde [Internet]. 2006 [access on 11/02/2019];8(31):63-70. Available at: http://cqh.org.br/portal/ pag/anexos/baixar.php?p_ndoc=224\&p_nanexo=66

5. Jericó MC, Perroca MG, Penha VC. Mensuração de indicadores de qualidade em centro cirúrgico: tempo de limpeza e intervalo entre cirurgias. Rev Latino-Am Enferm. 2011;19(5):1-8. http://dx.doi. org/10.1590/S0104-11692011000500023

6. Bogh SB, Falstie-Jensen AM, Hollnagel E, Holst R, Braithwaite $J$, Johnsen SP. Improvement in quality of hospital care during accreditation: a nationwide stepped-wedge study. Int J Qual Health C. 2016;28(6):715-20. https://doi.org/10.1093/intqhc/mzw099

7. Braga A, Pena MM, Melleiro MM. Métrica de indicadores assistenciais de hospitais certificados. Rev Enferm UFPE Online. 2018;12(3):66575. https://doi.org/10.5205/1981-8963-v12i3a230715p665-675-2018

8. Dalcól C, Garanhani ML. Papel gerencial do enfermeiro de centro cirúrgico: percepções por meio de imagens. Rev Eletr Enf. 2016;18:e1168. http://dx.doi.org/10.5216/ree.v18.34888

9. Ribeiro HCTC, Rodrigues TM, Teles SAF, Pereira RC, Silva LLT, Mata LRF. Distrações e interrupções em sala cirúrgica: percepção de profissionais de enfermagem. Esc Anna Nery. 2018;22(4):e20180042. http://doi.org/10.1590/2177-9465-EAN-2018-0042

10. Costa Junior AS, Leão LEV, Novais MAP, Zucchi P. An assessment of the quality indicators of operative and non-operative times in a public university hospital. Einstein. 2015;13(4):594-9. http://dx.doi. org/10.1590/S1679-45082015GS3289

11. Brasil. Ministério da Saúde. Portaria n 2.616, de 12 de maio 1998. Programa de controle de infecções hospitalares [Internet]. Brasília: Diário Oficial da União, 1998 [access on 09/26/2019]. Available at: http://bvsms.saude.gov.br/bvs/saudelegis/gm/1998/ prt2616_12_05_1998.html

12. Galvão TF, Lopes MCC, Oliva CCC, Araújo MEA, Silva MT. Patient safety culture in a university hospital. Rev Latino-Am Enferm. 2018;26:e3014. http://dx.doi.org/10.1590/1518-8345.2257.3014

13. Elias ACGP, Schmidt DRC, Yonekura CSI, Dias AO, Ursi ES, Silva RPJ, et al. Avaliação da adesão ao checklist de cirurgia segura.
Rev SOBECC. 2015;20(3):128-33. http://dx.doi.org/10.5327/ Z1414-4425201500030002

14. Marquioni FSN, Moreira TR, Diaz FBBS, Ribeiro L. Adesão ao checklist de cirurgia segura. Rev SOBECC. 2019;24(1):22-30. https://doi. org/10.5327/10.5327/Z1414-4425201900010006

15. Martins FZ, Dall'Agnol CM. Centro cirúrgico: desafios e estratégias do enfermeiro nas atividades gerenciais. Rev Gaúcha Enferm. 2016;37(4):e56945. http://dx.doi.org/10.1590/1983-1447.2016.04.56945

16. Pinto EV, Lunardi LS, Treviso P, Botene DZA. Atuação do enfermeiro na cirurgia robótica: desafios e perspectivas. Revista SOBECC. 2018;23(1):43-51. https://doi.org/10.5327/Z1414-4425201800010008

17. Al-Sughayir MA. Effect of accreditation on length of stay in psychiatric inpatients: pre-post accreditation medical record comparison. Int J Ment Health Syst. 2016;10(1):55. https://dx.doi. org/10.1186\%2Fs13033-016-0090-6

18. Shaw CD, Groene O, Botje D, Sunol R, Kutryba B, Klazinga N, et al. The effect of certification and accreditation on quality management in 4 clinical services in 73 european hospitals. Int J Qual Health C. 2014;26(Supl. 1):100-7. https://doi.org/10.1093/intqhc/mzu023

19. Gabriel CS, Bogarin DF, Mikael S, Cummings G, Bernardes A, Gutierrez $L$, et al. Perspectiva dos enfermeiros brasileiros sobre o impacto da acreditação hospitalar. Enferm Glob [Internet]. 2018 [access on 09/27/2019];17(49):381-419. Available at: http://scielo.isciii.es/scielo. php?script=sci_arttext\&pid=S1695-61412018000100381 \&lng=pt\&n $\mathrm{rm}=$ iso\&tlng=en

20. Gözlü K, Kaya S. Patient safety culture as perceived by nurses in a Joint Commission International Accredited Hospital in Turkey and its comparison with Agency for Healthcare Research and Quality Data. Patient Saf Qual Improv [Internet]. 2016 [access on 09/27/2019];4(4):440-9. Available at: http://psj.mums.ac.ir/ article_7640_9f9dd2489fbe1d50566a94452298fccc.pdf

21. Carrillo I, Mira JJ, Vicente MA, Fernandez C, Guilabert M, Ferrús L, et al. Design and testing of BACRA, a Web-Based Tool for middle managers at health care facilities to lead the search for aolutions to patient safety incidents. J Med Internet Res [Internet]. 2016 [access on 05/22/2020];18(9):e257. Available at: http://www.jmir.org/2016/9/ e257/. http://doi.org/10.2196/jmir.5942 